\title{
Influence of Laser Activating Nutrient Media on Bacterial Growing Dynamics
}

\author{
Lubov A. Kokorina, Anna V. Neupokoeva*, Elena R. Gerok, and Ksenia V. Rogova \\ Irkutsk State Medical University, Department of Microbiology, Virology and Immunology, Department of Medical and \\ Biological physics, 1 Krasnogo Vosstaniya str., Irkutsk 664009, Russian Federation
}

*e-mail: annett2005@inbox.ru

\begin{abstract}
The laser irradiation effect of nutrient media solutions on their microstructure and, as a result, on the dynamics of bacterial growth in photomodified media is considered in the article. It is experimentally shown that the growth rate of bacteria varies depending on the irradiation media time, that allows us to control the dynamics of bacterial cultures. (C) 2020 Journal of Biomedical Photonics \& Engineering.
\end{abstract}

Keywords: laser biostimulation; structure modification.

Paper \#3347 received 16 Jan 2020; revised manuscript received 17 Feb 2020; accepted for publication 7 Mar 2019; published online 12 Mar 2020. doi: 10.18287/JBPE20.06.010301.

\section{Introduction}

Laser radiation is widely used to modify a structure and properties of biomaterials, drugs, and biological fluids [1-4]. In the case of exposure to a liquid media, the energy of laser radiation is used to modify a microstructure of the liquid. It is known $[5,6]$ that organic molecules (for example, proteins macromolecules) and water molecules themself form clusters using hydrogen bonds. The larger size of such associate means the higher ratio of its mass to the surface and, hence, the smaller reactivity. It is shown $[4,7]$ that under the influence of laser radiation clusters of organic molecules can break up into smaller formations, because coherent monochromatic laser radiation causes intense movements of charged sections of macromolecules. As a result, the molecules break out of the clusters, creating more homogeneous mixture with fewer inhomogeneities.

On the other hand, the control of bacterial cultures growth is an urgent problem in medicine, because traditional methods of bacterial reproduction suppressing, for example, with the help of antibiotics, are not always effective. Therefore, some strains of Staphylococcus aureus have become resistant to a wide range of antibiotics, in particular to penicillins (methicillin, dicloxacillin, nafcillin, oxacillin, etc.) and cephalosporins. Resistance of bacteria leads to various complications after staphylococcal infection and causes high mortality. Therefore, the search for new methods for control of the bacterial growth dynamics is an urgent task.

The dynamics of bacterial culture development depends to a significant extent on both the presence of nutrients and their bioavailability. Therefore, there are three stages of cultivation for any bacterial culture in an in vitro closed environment: rapid growth under nutrients excess, "plateau" - the period when the processes of reproduction and death balance each other, and the decline period, when the depletion of nutrients leads to the predominance of the death processes over the growth processes of the culture. It is known that liquid or gel protein solutions are often used as a nutrient medium for the microorganisms cultivation [8], for example, meat-peptone agar. As noted above, dissolved substances form clusters (associates) in liquids by combining molecules. The associate size affects its diffusion through the bacterial membrane and, thus, its bioavailability as a food for bacterial cells. Therefore, the destruction of the molecule clusters, for example, under the influence of laser radiation, would allow us to change their suitability for assimilation by microorganisms.

The aim of this work is to determinate the effect of modification of the nutrient medium by laser irradiation on the growth dynamics of microorganisms in a closed system.

\section{Materials and methods}

The influence of nutrient medium laser exposure on the bacterial growth dynamics was evaluated. As a test model, we used a reference culture of Staphylococcus aureus (St. aureus) that was grown in a liquid medium (nutrient broth is an aqueous solution of peptone, fish meal hydrolysate, and sodium chloride). In the control cases, a microbial suspension was added to the liquid nutrient medium immediately after its preparation. In 
experimental samples, the nutrient medium that is necessary for the development of bacterial culture was exposed to laser radiation in the green region of the spectrum (wavelength $532 \mathrm{~nm}$, power $100 \mathrm{~mW}$ ). The choice of this wavelength is determined by the results of previous experiments on the effect of laser radiation on protein solutions [9]. Laser radiation with wavelength of $532 \mathrm{~nm}$, even under small exposure times, provides a larger change of the structure compared to the wavelength of $650 \mathrm{~nm}$.

The irradiation of the nutrient medium was carried out in the "light boiler" mode. Laser radiation was directed from above to the liquid nutrient medium in a test tube, which was wrapped with a reflective layer. Thus, the liquid medium was affected not by direct radiation only, but also by reflected radiation. Laser radiation was exposed during 30,60, and $120 \mathrm{sec}$ (the total irradiation energy is $3 \mathrm{~J}, 6 \mathrm{~J}$, and $12 \mathrm{~J}$, respectively). After that, a microbial suspension was placed in the nutrient medium. It should be noted that the laser radiation affected the nutrient medium only and did not interact with bacteria. Every 12-24 hours during the week, a drop of liquid medium with microbial suspension was taken and seeded on a solid agar-based medium in four repetitions (4 Petri dishes). Further, the primary growth time was taken into account, the number of colony-forming units (CFU) was calculated and their types were described. Thus, for each of the 8 time points, 4 seeding procedures were carried out, a total of 32 samples for each series of experiments, or 128 samples in total. The average value and measurement error were calculated from 4 measurements for each time point.

\section{Results}

The first growth of bacteria in the control and experimental groups was recorded 12 hours after sowing (Fig. 1). However, in a group with nutrient medium irradiated during $120 \mathrm{sec}$ growth was delayed: growth was observed only in one Petri dish.

There is a 24-hour active growth stage in the control group, where St. aureus was cultured in a non-laserexposed medium (Fig. 1). At the same time, the number of bacteria has its maximum $-7.925 \pm 0.15 \mathrm{lg} \mathrm{CFU} / \mathrm{ml}$. The number of cells decreases to $4.535 \pm 0.11$ $\lg \mathrm{CFU} / \mathrm{ml}$ from 24 hours point to the end of the experiment.

For the culture medium irradiated for 30 and $60 \mathrm{sec}$, the dynamics of the bacteria growth is identical to the control group in the first 24 hours. However, the medium irradiated for $30 \mathrm{sec}$ later shows a smoother decrease of the colonies number that is 1.32 times greater at the end of experiment than for the nonirradiated medium. The difference significantly exceeds the error of the experiment at this stage.

The irradiation of the media during $60 \mathrm{sec}$ increases the active breeding period of the microbial population to 72 hours. In addition, the population size reached its maximum value in comparison with all experimental cases $(7.75 \pm 0.5 \lg \mathrm{CFU} / \mathrm{ml})$. After that, the death processes began to predominate over the division processes (it is associated with the nutrient substrate depletion) and the number of bacteria decreased to $5.175 \pm 0.35 \mathrm{lg} \mathrm{CFU} / \mathrm{ml}$, that differs from the control value within the experimental error.

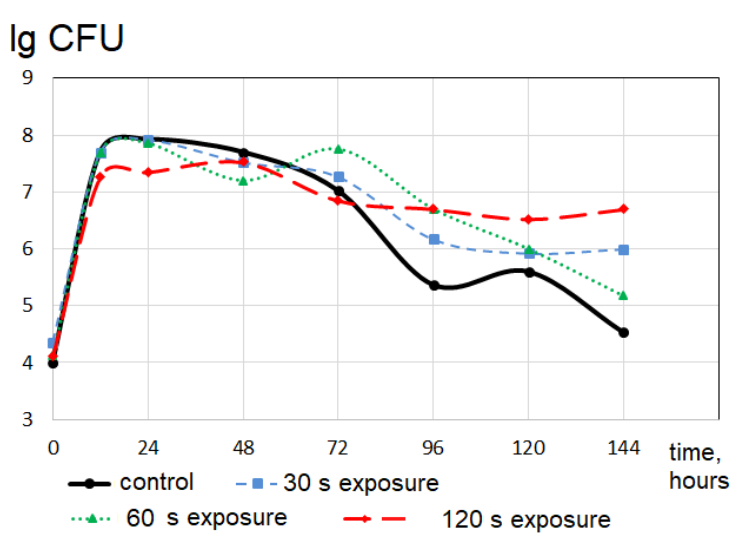

Fig. 1 The dependence of bacterial growth over the time (the abscissa axis sets time, the ordinate axis sets the logarithm of colony-forming units (CFU).

With an increase of the laser exposure time to $120 \mathrm{sec}$ the bacteria growth was slowed down at the initial stage compared to the control group. In this case, the active breeding phase of the microbial population was extended to 48 hours, followed by a slight decrease in the number of colonies. "Plateau" is registered until the completing experiment, so the processes of reproduction and death are approximately at the same level. It should be noted that the absence of bacteria amount reducing phase leads to the fact that the amount of colonies in the $120 \mathrm{sec}$ exposure media exceeds the control value by 1.5 times to the end of the experiment.

The observed significant difference in growth dynamics is probably due to changes in the structure of the macromolecular clusters in the nutrient medium. Thus, at short exposure times ( 30 and $60 \mathrm{sec}$ ) the laser radiation energy is spent on increase of the cluster components mobility that leads to its disintegration into smaller formations and increase of the nutrients bioavailability in the solution. These changes cause an elongation of the bacteria growth phase and a sharp depletion of nutritional resources, that later leads to intensive bacterial death.

Under irradiating the nutrient medium during $120 \mathrm{sec}$, we observe the opposite effect: at the initial stage, the bacteria growth rate was less than control, and this fact indicates a decrease of the nutrients bioavailability. We offer two explanations for this phenomenon. First, under the excess of the laser radiation energy, additional energy is enough not only to destroy clusters but also to change the secondary and tertiary conformation of protein molecules that negatively affects their interaction with receptors on the bacterial membrane. Second, the explanation may be since according to some experimental data [10] laser radiation causes an increase in the homogeneity of the 
liquid media structure, i.e., there are two parallel processes: division of the large clusters into smaller ones, and on the contrary, fusion, enlargement of the smallest clusters. Thus, the dispersion degree of the clusters size decreases, while the percentage of the smallest ones decreases, which also causes a bacteria growth slowdown in the media and increases the time until the resources are depleted, that looks like a long "plateau" on the graph.

\section{Conclusion}

Thus, it is experimentally established that laser irradiation with a wavelength of $532 \mathrm{~nm}$ significantly affects growth dynamics of the Staphylococcus aureus in a closed system. Nutrient media irradiated for 30 and
$60 \mathrm{sec}$ contribute to a longer growth period compared to the control that is probably associated with decrease in the characteristic size of clusters formed by macromolecules of nutrients that increases their bioavailability. Irradiation of the nutrient medium during $120 \mathrm{sec}$ leads to the opposite effect: the rate of the bacteria growth slows down, the consumption of the nutrient resources decreases, and the phase of active death does not occur by the end of the observation time.

\section{Disclosures}

All authors declare that there is no conflict of interests in this paper.

\section{References}

1. G. E. Brill, A. V. Egorova, I. O. Bugaeva, and G. V. Ponomarev, "Laser modification of the dehydration selforganization of the dimegin hydrophilic photosensitizer," Laser medicine 15(2), 59-60 (2011) [in Russian].

2. S. Kudryashov, Yu. Kolobov, and A. Ligachev, "Multiscale femtosecond laser texturing and chemical modification of the surface of medical titanium implants," Photonics 3(45), 14-22 (2014) [in Russian].

3. T. I. Karu, V. I. Yusupov, V. N. Bagratashvili, A. L. Drozdov, and V. M. Chudnovskii, "Influence of lowintensity red diode and laser radiation on the locomotor activity of sea urchin sperm," Doklady Biochemistry and Biophysics 457(1), 146-148 (2014).

4. A. N. Malov, A. Yu. Seteikin, A. V. Neupokoeva, E. S. Musatova, I. E. Golub, L. V. Sorokina, V. S. Fetschenko, and A. A. Vaichas, "The laser radiation action on the biological objects," Optik 124(23), 60346041 (2013).

5. E. A. Manykin, V. B. Oshurko, "Water ordering under laser radiation," Laser Physics 17(6), 842-852 (2007).

6. V. D. Lakhno, Clusters in Physics, Chemistry, Biology, NITs "Regular and chaotic dynamics", Izhevsk, Russia (2001) [in Russian].

7. A. N. Malov, A. A. Vaichas, Laser Control of Biomineral Formation. Physical Principles and Prospects of Developing New Medical Nanotechnology, Palmarium Academic Publishing, Saarbrucken, Deutschland (2016) [in Russian]. ISBN 978-3-659-60476-8.

8. N. V. Prozorkina, L. A. Rubashkina, Fundamentals of Microbiology, Virology and immunology, Phoenix, Rostov na Donu, Russia Federation (2002) [in Russian]. ISBN 5-222-02499-7.

9. A. N. Malov, S. A. Nebogin, A. V. Neupokoeva, and A. A. Vaychas, "Probe microscopy of biological fluid crystallograms after laser impact," Journal of Biomedical Photonics \& Engineering 4(1), 010405 (2018).

10. A. V. Neupokoeva, E. A. Kuzina, N. S. Moskalev, A. I. Nikonova, and S. A. Nebogin, "Laser photomodification of insulin solution," Journal of Biomedical Photonics \& Engineering 5(2), 20301 (2019). 